\title{
Fault Diagnosis for Constant Deceleration Braking System of Mine Hoist based on Principal Component Analysis and SVM
}

\author{
Juan-Juan $\mathrm{Li}^{1}$, Liang Hu${ }^{1}$, Guo-Ying Meng ${ }^{1, a}$, Guang-Ming Xie ${ }^{2}$, Ai-Ming Wang ${ }^{1}$, Shuai Wang ${ }^{1}$ and Yi-Fan Jia ${ }^{1}$ \\ ${ }^{1}$ School of Mechanical Electronic and Information Engineering, China University of Mining and Technology (Beijing), Beijing 100083, China \\ ${ }^{2}$ College of Engineering, Peking University, Beijing 100871, China
}

\begin{abstract}
Based on AMESim simulation platform, the pressure-time curve of constant deceleration braking system is obtained in this paper firstly, by simulating three typical faults of brake, the spring stiffness decrease, the brake shoe friction coefficient decrease and brake leaking. Then pressure data on the curve for each time are seen as a variable and the curve is chosen as the fault sample, analysed by the method of Principal Component Analysis (PCA). Last, principal components or sum of variance contribution rates more than $95 \%$ are selected as sample eigenvalues and Support Vector Machine (SVM) is used for fault diagnosis. Diagnosis results show that all testing faults can be identified accurately, which indicates SVM model has an extremely excellent ability to identify faults. To further verify the performance of SVM for fault identification, BP neural network is established to compare. The result shows that SVM model is more accurate than BP neural network in fault recognition.
\end{abstract}

\section{Preface}

As the key equipment in the production process, connection between the mine ground and underground, the mine hoist undertakes the important task to lift coal or ore, down materials and elevate personnel or equipment ${ }^{1}$. Braking system of the hoist is the final barrier to ensure safe operation. Its braking performance directly affects safety and reliability of hoist. Thus, fault diagnosis of the brake has become a hot spot of research.

According to uncertain problems in the fault diagnosis of hoist, the literature ${ }^{2}$ proposes a fault diagnosis method based on ontology and Bayesian network. The literature ${ }^{3}$ extracts feature vectors by wavelet packet of 3 layers and reconstruction method, takes results of neural network recognition as independent evidence, and judges based on evidence theory of synthesis. The literature ${ }^{4}$ presents a multi-class classification algorithm based on ordering binary tree SVM for fault diagnosis. Also, there are other methods for fault diagnosis like SVM ${ }^{5}$, the genetic algorithm to optimize the BP neural network ${ }^{6}$, SOM neural network method ${ }^{7}$, particle swarm optimization ${ }^{8}$ and so on. Effective results of fault diagnosis can be gotten by adapting methods mentioned above on some certain conditions, but in those methods, there are still some problems that parameters can't be accurately gotten, or can't represent the general characteristics of the braking system ${ }^{9}$. For example, online monitoring of the friction coefficient still has no mature feasible solution ${ }^{10}$; the signal linearity and zero drift problem of brake pressure sensors are difficult to solve because of its long-term load. In order to overcome deficiencies of methods mentioned above, this paper presents a brand-new method which extracts principal components from pressure-time curve of the brake system as characteristic parameters and uses the method SVM for fault diagnosis.

\section{Principal Component Analysis}

Principal Component Analysis (PCA) adapts the thought of dimension reduction which converts multiple index to a few comprehensive index. PCA is a statistical procedure that uses an orthogonal transformation to convert a set of observations of possibly correlated variables into a set of values of linearly uncorrelated variables called principal components, keeping the total variance of variables constant during the transformation. This transformation is defined in such a way that the first principal component has the largest variance, the second principal component corresponding to the second largest variance, and so on. The way of saving lower order principal component and ignoring the higher can maintain the main features of data, which can achieve the purpose of dimension reduction. The flowchart of principal component analysis is shown in Figure 1.

a Guo-Ying Meng: mgy@cumtb.edu.cn 


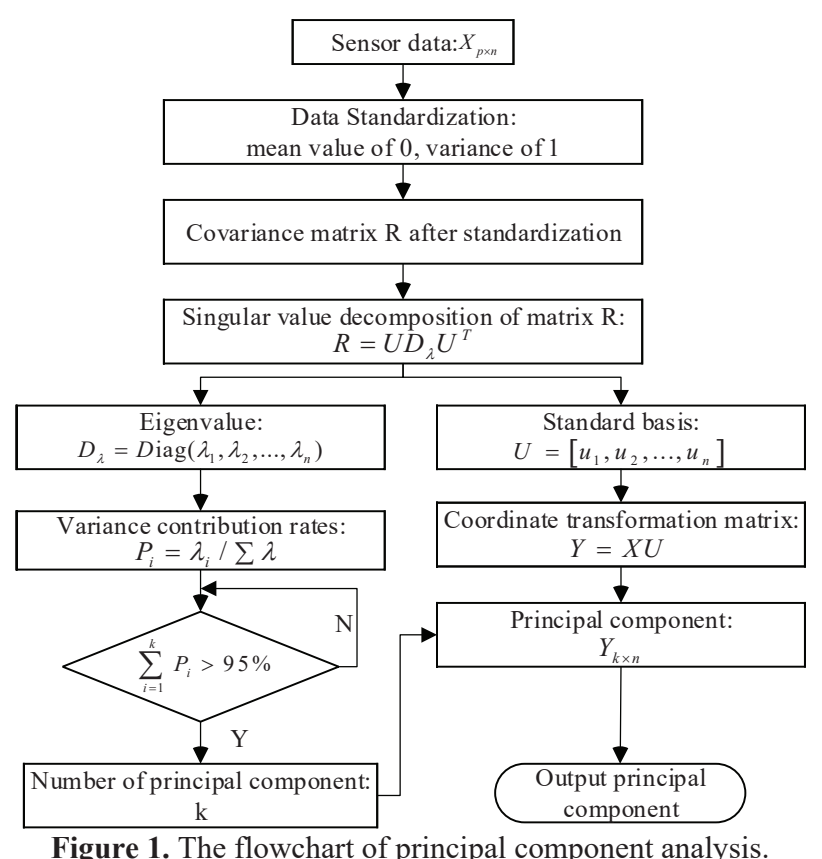

\section{Support Vector Machine}

\subsection{Introduction of Support Vector Machine}

Support Vector Machine (SVM), a machine learning algorithm, is developed on the basis of the statistical theory. The main idea of SVM is to establish a classification hyperplane, maximizing two types of intervals. SVM is the approximate realization of the structural risk minimization principle.

When linear classification, the classification face, $(w \cdot x)+b=0$, is defined on the place farthest away from two groups of samples. Then the solving optimal hyperplane problem can be transformed into its dual problem by using the Lagrangian optimization technique, or

$$
\left\{\begin{array}{lr}
\max Q(a)=\sum_{i}^{I} \alpha_{i}-\frac{1}{2} \sum_{i=1}^{I} \sum_{j=1}^{I} \alpha_{i} \alpha_{j} y_{i} y_{j}\left(x_{i} x_{j}\right) \\
\text { s.t. } \sum_{i=1}^{I} \alpha_{i} y_{j}=0, & \alpha_{i} \geq 0
\end{array}\right.
$$

Where $\left(x_{i}, y_{i}\right)$ is the sample needed to classify; I is the number of samples; $\alpha_{i} \geq 0(i=1,2, \ldots, I)$ is Lagrange multiplier.

Equation (1) is the quadratic function optimization subject to an inequality. Suppose its optimum solution is $\mathrm{X}_{\mathrm{p} \times \mathrm{n}}$ (sensor data), the optimal $\mathrm{W}^{*}$ and classification threshold $b^{*}$ could be found. The optimal classification function, Equation (2), can be found.

$$
f(x)=\operatorname{sgn}\left[\sum_{i=1}^{I} \alpha_{i}^{*} y_{i}\left(x_{i}\right)+b^{*}\right]
$$

When nonlinear classification, linearly inseparable samples in lower dimensional space are usually mapped to the linearly separable space of higher dimension, where the optimal hyperplane is constructed. The way of constructing the optimal hyperplane in the higher dimensional space is similar to linearly separable SVM, that is, dot product in the higher dimensional space is replaced by kernel function $\mathrm{K}\left(\mathrm{x}_{\mathrm{i}}, \mathrm{x}_{\mathrm{j}}\right)$ subject to Mercer. Finally, the optimal classification function is the Equation (3).

$$
\begin{aligned}
& f(x)=\underset{i}{\operatorname{sgn}}\left[\mathrm{W}^{*} \emptyset(\mathrm{x})+\mathrm{b}^{*}\right] \\
& =\operatorname{sgn}\left[\sum_{\mathrm{i}=1}^{\mathrm{I}} \alpha_{\mathrm{i}}^{*} \mathrm{y}_{\mathrm{i}} \mathrm{K}\left(\mathrm{x}_{\mathrm{i}}, \mathrm{x}\right)+\mathrm{b}^{*}\right]
\end{aligned}
$$

The flowchart of SVM is shown in the Figure 2.

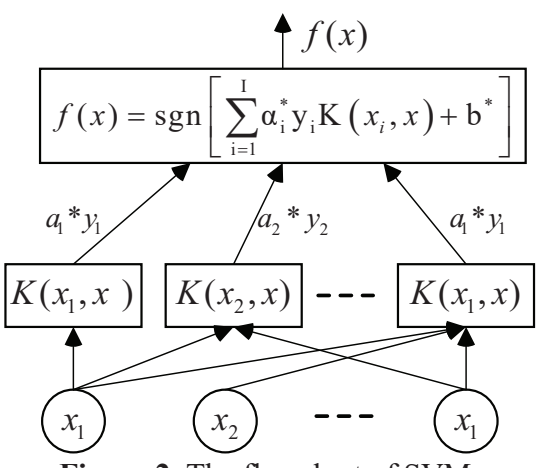

Figure 2. The flowchart of SVM.

\subsection{Support Vector Machine Multiclass Classification}

Support Vector Machine is originally designed for binary classification problems, but when processing multiclass classification problems, the SVM multiclass classifier is needed to construct. There are two common ways to construct the SVM multiclass classifier, direct method and the indirect. Direct method is to directly modify the object function, combining parameter solution of multiple classification faces into an optimization problem, by solving the optimization problem, "one time" to realize classification. Because of the high computational complexity, the high difficulty to realize, direct method is only suitable for simple problems. Indirect method is mainly to realize construction of multiple classifiers by combining multiple binary classifiers. The common ways of indirect method are "one versus one (1-V-1)", "one versus rest (1-V-R)" and Hierarchical Support Vector Machine (H-SVM).

\subsection{1 "1-V-1" Multiclass Classification}

One SVM classifier is needed to be designed between any two groups of samples, so k(k-1)/2 classifiers are needed for k-class samples. After classifying an unknown sample $\mathrm{x}$ by $(\mathrm{k}-1) / 2$ classifiers, which category the unknown sample $\mathrm{x}$ belongs to is defined by which the largest number of votes in k-class samples.

\subsection{2 “1-V-R” Multiclass Classification}

Classifying a type of samples into one class, the rest into one another class, k SVM classifiers can be constructed to k-class samples. The unknown sample belongs to the 
class which has the maximum classification function value.

\subsubsection{Hierarchical Support Vector Machine}

$\mathrm{H}-\mathrm{SVM}$ is the method that firstly all categories are divided into two subclasses, and then the subclass is further divided into two secondary subclasses, and so on, until a separate category so far.

\section{Fault Simulation and Diagnosis}

In the working process of mine hoist, the performance of its brake inevitably degenerates gradually until the brake fails, because of its brake springs, brake cylinders, pistons and rings working under the long-term high load condition. In this paper, we will simulate and diagnose three typical faults of brake, the spring stiffness decrease, the brake shoe friction coefficient decrease and brake leaking. The flowchart of fault simulation and diagnosis is shown in Figure 3.

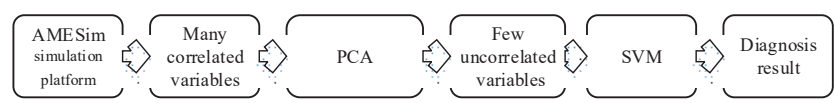

Figure 3. The flowchart of fault simulation and diagnosis.

\subsection{Simulating Samples of Faults}

Taking the matching constant deceleration braking system JKMD4.5X4 of mine hoist E141A as the research object, a fault simulation model of the system is built based on AMESim simulation platform ${ }^{10}$. Only considering the overloaded working condition, simulation time is set to $10 \mathrm{~s}$, including $1-2 \mathrm{~s}$ for energy accumulator charging, $2-5 \mathrm{~s}$ for brake releasing, 5-8s for constant deceleration braking, and $8-10 \mathrm{~s}$ for system pressure relief; besides, the sampling frequency is set to $100 \mathrm{HZ}$. Pressure data from 5-8.2s are collected as a group of fault data; besides, each type of faults is simulated by 30 groups of data. The pressure-time curve of the brake system is gotten by simulating three typical faults, as is shown respectively in the Figure 4, Figure 5 and Figure 6.

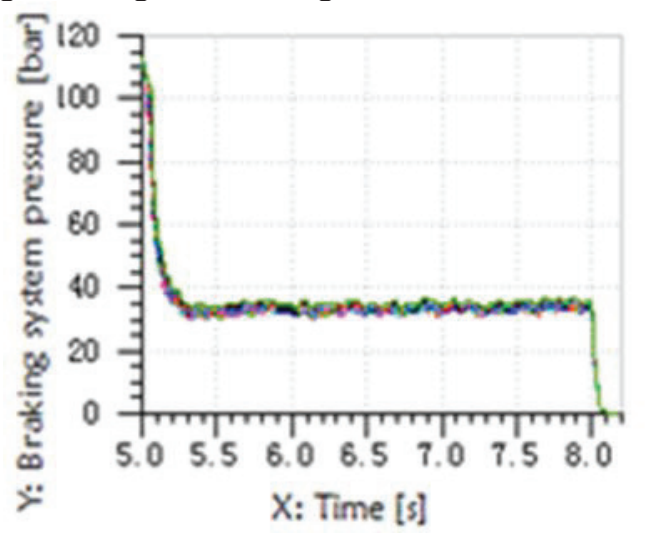

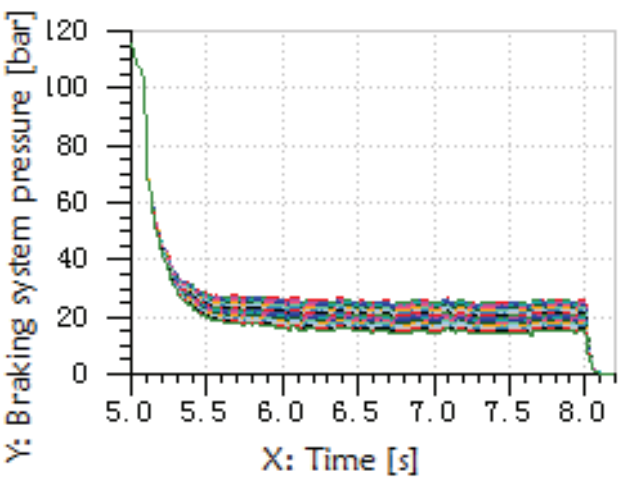

Figure 5. Braking system pressure in different brake shoe friction coefficient.

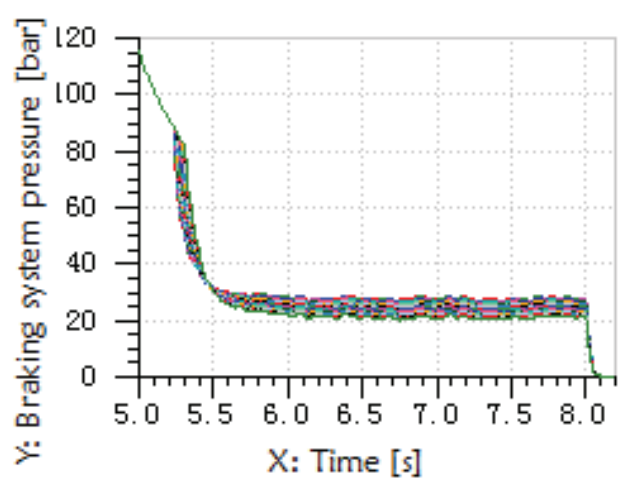

Figure 6. Braking system pressure in different leakage.

\subsection{Extracting Sample Eigenvalues of Fault Samples}

From 30 groups of data, 25 groups are selected randomly for training samples, the remaining 5 groups for test samples. Thus, for three typical faults, there are 75 groups of 320-dimensional (acquisition time of 3.2s, sampling frequency of $100 \mathrm{HZ}$ ) training data and 15 groups of 320-dimensional test data. Data are seen as a variable in each dimension, which means each group of data can be viewed as the sample consisting of 320 variables, then analysed by the method of PCA. Besides, principal components or sum of variance contribution rates more than $95 \%$ are selected as sample eigenvalues. In this paper, the first five principal components are selected as eigenvalues. After normalization processing, eigenvalues and fault types from part of training samples and test samples are shown respectively in the Table 1 and Table 2.

Figure 4. Braking system pressure in different spring stiffness.

Table 1. SVM training samples.

\begin{tabular}{|c|c|c|c|c|c|c|c|}
\hline \multirow{2}{*}{$\begin{array}{l}\text { Sample } \\
\text { number }\end{array}$} & \multicolumn{5}{|c|}{ SVM training input } & \multirow{2}{*}{$\begin{array}{c}\text { SVM } \\
\text { training } \\
\text { output }\end{array}$} & \multirow{2}{*}{ Corresponding fault } \\
\hline & P1 & P2 & P3 & P4 & P5 & & \\
\hline
\end{tabular}




\begin{tabular}{|c|c|c|c|c|c|c|c|}
\hline 1 & 0.6893 & -0.9687 & -0.8108 & 0.1625 & -0.8115 & -1 & \multirow{3}{*}{$\begin{array}{c}\text { The brake shoe friction } \\
\text { coefficient decrease }\end{array}$} \\
\hline 2 & 0.7704 & -0.8557 & -0.5879 & 0.1314 & 0.1379 & -1 & \\
\hline 3 & 0.6777 & -0.9845 & -0.8408 & 0.1659 & -0.9119 & -1 & \\
\hline$\ldots$ & $\ldots$ & $\ldots$ & $\ldots$ & $\ldots$ & $\ldots$ & $\ldots$ & \multirow{4}{*}{$\begin{array}{l}\text { The spring stiffness } \\
\text { decrease }\end{array}$} \\
\hline 26 & -0.1823 & 0.9381 & -0.8849 & -0.6818 & 0.1901 & 0 & \\
\hline 27 & 0.0784 & 0.7384 & -0.4693 & 0.3522 & 0.3064 & 0 & \\
\hline 28 & 0.0335 & 0.7671 & -0.5333 & 0.212 & 0.2971 & 0 & \\
\hline$\ldots$ & $\ldots$ & $\ldots$ & $\ldots$ & $\ldots$ & $\ldots$ & $\ldots$ & \multirow{4}{*}{ Brake leaking } \\
\hline 73 & -0.6325 & -0.2598 & 0.9486 & 0.0567 & 0.1852 & 1 & \\
\hline 74 & -0.6726 & -0.2671 & 0.954 & 0.0562 & 0.1787 & 1 & \\
\hline 75 & 0.1868 & -0.0968 & 0.8477 & 0.0295 & 0.2844 & 1 & \\
\hline
\end{tabular}

Table2. SVM test samples.

\begin{tabular}{|c|c|c|c|c|c|c|}
\hline \multirow{2}{*}{$\begin{array}{l}\text { Sample } \\
\text { number }\end{array}$} & \multicolumn{5}{|c|}{ SVM test input } & \multirow{2}{*}{ Corresponding fault } \\
\hline & $\mathbf{P 1}$ & $\mathbf{P 2}$ & P3 & P4 & P5 & \\
\hline 1 & 0.8283 & -0.7775 & -0.4394 & 0.1114 & 0.705 & \multirow[b]{2}{*}{$\begin{array}{l}\text { The brake shoe friction } \\
\text { coefficient decrease }\end{array}$} \\
\hline 2 & 0.8166 & -0.7911 & -0.4609 & 0.1128 & 0.6902 & \\
\hline$\cdots$ & $\ldots$ & $\ldots$ & $\ldots$ & $\ldots$ & $\ldots$ & \multirow{4}{*}{ The spring stiffness decrease } \\
\hline 6 & -0.1615 & 0.92 & -0.8506 & -0.5873 & 0.2053 & \\
\hline 7 & 0.0113 & 0.7816 & -0.5654 & 0.1365 & 0.29 & \\
\hline$\ldots$ & $\ldots$ & $\ldots$ & $\ldots$ & $\ldots$ & $\ldots$ & \\
\hline 11 & -0.1708 & -0.1709 & 0.8906 & 0.0504 & 0.2495 & \multirow{2}{*}{ Brake leaking } \\
\hline 12 & -0.208 & -0.1783 & 0.8951 & 0.0518 & 0.2451 & \\
\hline
\end{tabular}

\subsection{Construction of the SVM classifier and testing results}

In this paper, the toolbox called "libsvm" is used for SVM fault diagnosis, which is developed by the C.J Lin, the professor of National Taiwan University. Because the type of kernel function and model parameters have a great impact on model performance, RBF kernel function, most widely used in SVM, is chosen in this paper, using cross-validation method for seeking the optimum parameter $\mathrm{c}$ (penalty factor) and parameter $\mathrm{g}$ (variance of RBF kernel function), then training model by the optimum parameter. After completing the training, the data are inputted for test and then the test result is gotten as is shown in the Figure 7.

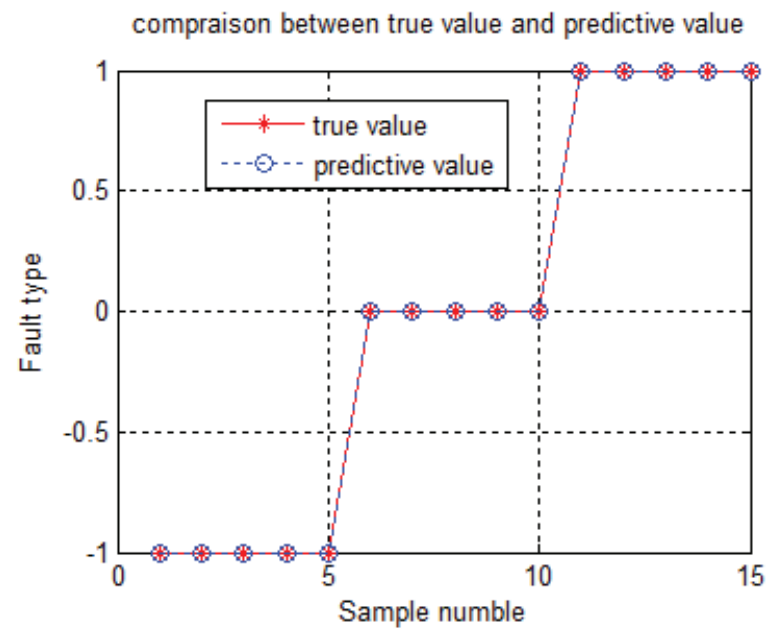

Figure 7. The result of SVM diagnosis.
As can be seen from the Figure 7, all testing faults can be identified accurately, the mean square error of 0 , which indicates SVM model has an extremely excellent ability to identify the fault.

To further verify the performance of SVM for fault identification, BP neural network is established to compare ${ }^{11}$. The result of BP neural network diagnosis is shown in the Figure 8.

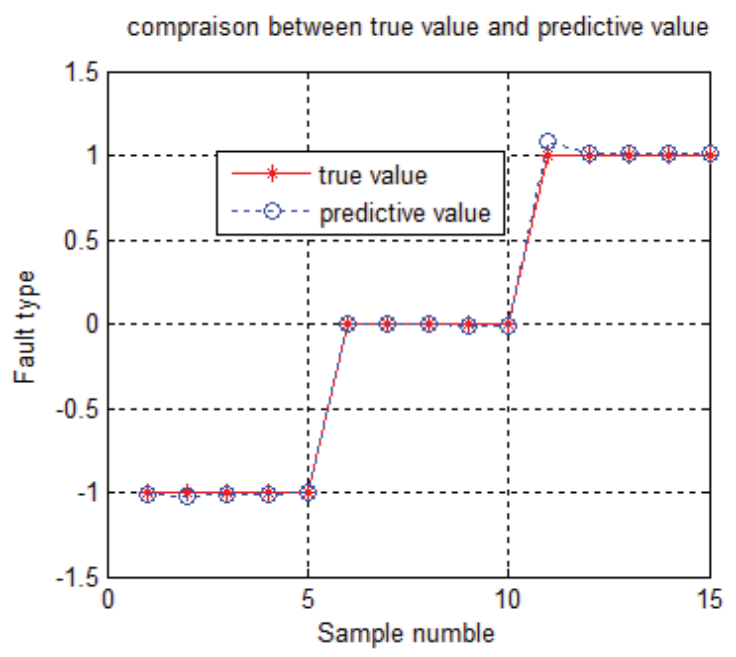

Figure 8. The result of BP neural network diagnosis.

As can be seen from the Figure 8, the diagnosed fault of 1-5 samples is the friction coefficient decrease, 6-10 samples for the spring stiffness decrease, 11-15 samples for brake leaking, which indicates diagnosed faults are consistent with testing faults. The BP neural network diagnosis also can identify the fault, but its mean square error of 0.0025 . That is, SVM model is more accurate than BP neural network in fault recognition. 


\section{Conclusion}

Based on AMESim simulation platform, the pressure-time curve of constant deceleration braking system is obtained in this paper firstly, by simulating three typical faults of brake, the spring stiffness decrease, the brake shoe friction coefficient decrease and brake leaking. Then pressure data on the curve for each time are seen as a variable and the curve is chosen as the fault sample, analysed by the method of Principal Component Analysis (PCA). Last, principal components or sum of variance contribution rates select more than $95 \%$ are selected as sample eigenvalues and Support Vector Machine (SVM) is used for fault diagnosis. Diagnosis results show that all testing faults can be identified accurately, which indicates SVM model has an extremely excellent ability to identify the fault. To further verify the performance of SVM for fault identification, BP neural network is established to compare. The result shows that SVM model is more accurate than BP neural network in fault recognition.

\section{Funding}

The authors are grateful to the National key research and development plan, "Basic theory and key technology of coal mine deep well construction and hoist". (Grant No.2016YFC0600900)

\section{References}

1. Bo Du, Bu-Bin Zhang, Hai-Ping Feng, Mining \& Processing Equipment, 44, 1-7 (2016)

2. Juan-Li Li, Zhao-Jian Yang, Journal of Vibration, Measurement \& Diagnosis, 33, 993-997 (2013)

3. Zheng-You Wang, Ji-Lin Liu, Journal of China Coal Society, 28,650-654 (2003)

4. Ying Wang, Ya-Li Gao, Jian-Wei Ma, Mining \& Processing Equipment, 39, 46-48(2011)

5. Xiao-Hui Guo, Xiao-Ping Ma, Journal of China University of Mining \& Technology, 35, 813-817 (2006)

6. Qiang Zhang, Nan Hu, Hong-Feng Li. Journal of Liaoning Technical University(Natural Science), 35, 155 -159 (2016)

7. Geng-Yun Zhang, Mining \& Processing Equipment, 39, 48-52 (2011)

8. Jing-Yan Liu, Fu-Zhong Wang, Yu-Dong Li, Control Engineering of China, 23, 294-298 (2016)

9. Rui Lian, Zheng-Guo Xu, Jian-Gang Lu, Safety in Coal Mines, 44, 131-133 (2013)

10. Juan-Juan Li, Liang $\mathrm{Hu}$, Guo-Ying Meng, Guang-Ming Xie et al, Industry and Mine Automation, 43, 55-60 (2017)

11. Juan-Juan Li, Ai-Ming Wang, Guo-Ying Meng, et al, Fault Diagnosis in Braking System of Mine Hoist Based on Monent Characteristics, (to be published) 